\title{
Wave-Function Reconstruction by Phase-Plate Transmission Electron Microscopy
}

\author{
B. Gamm*, M. Dries*, K. Schultheiss*, H. Blank*, A. Rosenauer**, R.R. Schröder***, and D.
} Gerthsen*

* Laboratorium für Elektronenmikroskopie, Karlsruher Institut für Technologie, D-76128 Karlsruhe, Germany

** Institut für Festkörperphysik, Universität Bremen, D-28359 Bremen, Germany

*** CellNetworks, BioQuant, Universität Heidelberg, D-69120 Heidelberg, Germany

Physical phase plates for transmission electron microscopy have been practically realized and applied to enhance contrast of weak-phase objects by generating an additional relative phase shift $\varphi_{p p}$ between scattered and unscattered electrons. Carbon-film-based Zernike- and Hilbert-phase plates [1,2] have been studied for some time already. We focus on electrostatic-potential-based approaches according to Boersch [3], which provide the possibility to control the phase shift by the applied voltage as an additional parameter.

While improvement of weak-phase object contrast is important for biological samples, the controlled change of phase contrast is also of interest for samples in materials science. The variation of $\varphi_{p p}$ can be exploited to reconstruct amplitude and phase of the image- and object-wave function. This was already recognized by Danev et al. [4] who suggested a reconstruction procedure by the use of a phase plate suited for weak-phase objects. We now propose a new technique for wave-function reconstruction, which is applicable for general objects and nonlinear image formation [5]. The procedure is based on only three images, which are taken with different and arbitrary $\varphi_{\mathrm{pp}}$ under otherwise identical conditions. Nonlinear and incoherent image contributions are effectively eliminated by the calculation of difference images, which are used for a full analytical reconstruction of the object-wave function of general objects.

To demonstrate the validity of the technique simulation of phase-contrast images and subsequent reconstruction from such images were carried out. Two different wave functions were used. An experimental object-wave function of platinum nanoparticles on amorphous carbon film was acquired by off-axis holography. Another object-wave function was calculated assuming a crystalline silicon sample with $50 \%$ vacancies in the atomic column indicated by an arrow. FIG. 1 and FIG. 2 show the original phase of the wave functions and the phase reconstructed from three simulated images using the new technique. The excellent agreement between the original and the reconstructed phase of the object-wave function is demonstrated more clearly in linescans (FIG. 1c) and FIG. 2c)) across a region of interest.

An ideal phase plate was assumed for the simulation of phase-contrast images. The influence of real phase plates must be considered e.g. the obstruction of electrons at certain spatial frequencies due to the phase-plate structure. The modification of the reconstructed wave function will then depend on the specific object and its spectrum of spatial frequencies. The investigation of the impact of noise on the reconstruction shows that even low electron doses do not significantly hamper the reconstruction process. 
References

[1] R. Danev et al., Ultramicroscopy 88 (2001) p243.

[2] R. Danev et al., J. Phys. Soc. Jpn. 73 (2004) p2718.

[3] K. Schultheiß et al., Rev. Sci. Instrum. 77 (2006) 033701.

[4] R. Danev et al., J. Phys. Soc. Jpn. 70 (2001) p696.

[5] B. Gamm et al., Ultramicroscopy, DOI: 10.1016/j.ultramic.2010.02.006

[6] The project is funded by the German Research Foundation (Deutsche

Forschungsgemeinschaft) under Ge 841/16 and Sch 424/11.
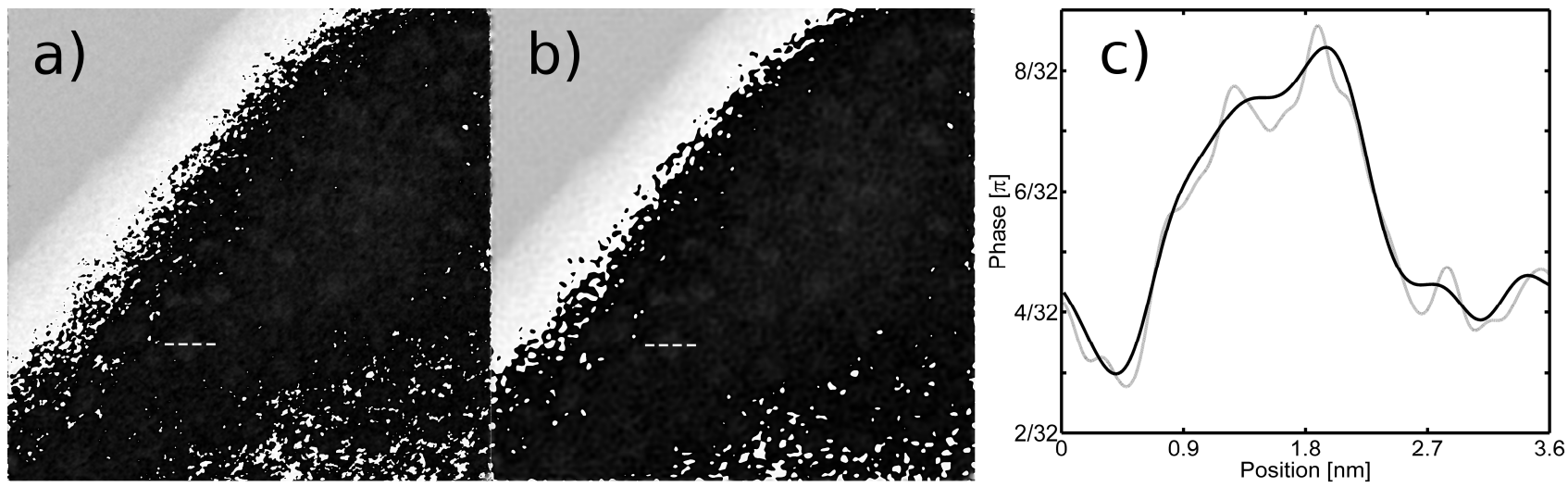

FIG. 1. a) Phase of the original wave function of Pt nanoparticles on amorphous carbon film acquired with off-axis electron holography (scale from $0 /$ black to $2 \pi$ /white). $b$ ) phase reconstructed from simulated phase plate TEM c) Linescans across a particle, taken from a) (gray line) and taken from $b$ ) (black line). The position of the line scan is marked in a) and b) by the white dashed line.
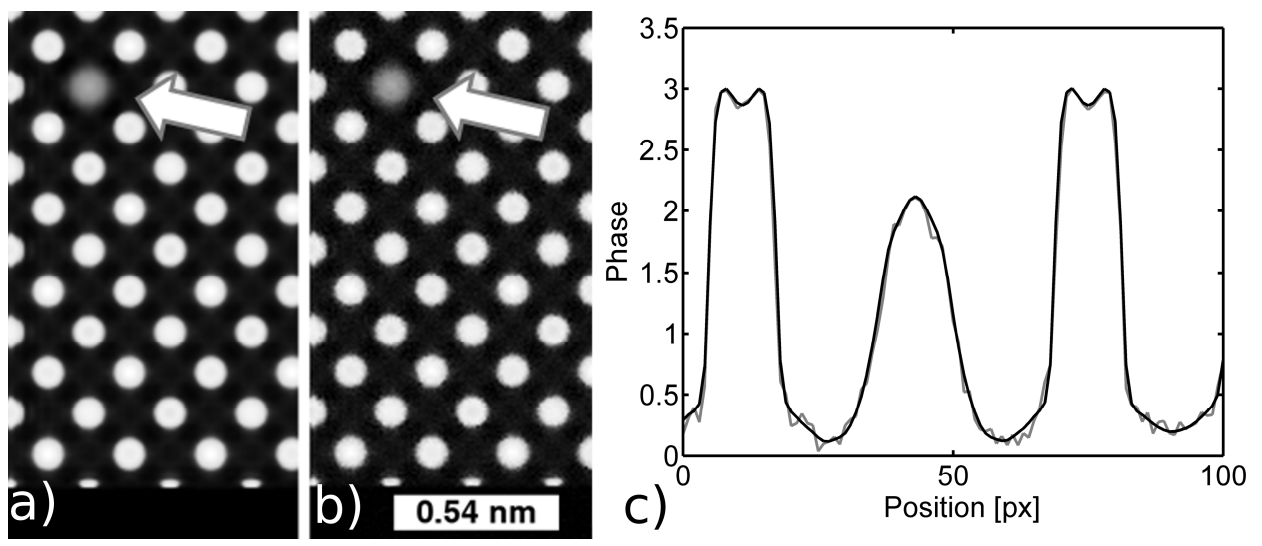

FIG. 2. a) Phase of the original simulated wave function representing a single crystalline silicon sample along the [100]-zone axis (sample thickness of $5.431 \mathrm{~nm}$ ) b) phase of the wave function reconstructed from simulated phase plate TEM. White arrows are pointing at an atomic column containing 50\% vacancies. c) Linescans across three atom positions. Black taken from a) across the vacancy position and two adjacent atom positions, and gray taken from $b$ ) at the same location. Apart from little noise (from noisy simulated images) the linescans are in very good agreement, and show that the reconstruction works even if non-linear contributions are present in the simulated images. 